\title{
Incidence and Comorbidity of Reactive Attachment Disorder: Based on National Health Insurance Claims Data, 2010-2012 in Korea
}

\author{
Minha Hong1, Duk Soo Moon², Hyejung Chang ${ }^{3}$, Seung Yup Lee ${ }^{4}$, Seong Woo Cho ${ }^{4}$, \\ Kyung-Sook Lee ${ }^{5}$, Jin-Ah Park ${ }^{6}$, Sang Min Lee ${ }^{7}$, and Geon Ho Bahn ${ }^{8} \bowtie$ \\ ${ }^{1}$ Department of Psychiatry, Seonam University College of Medicine, Myongji Hospital, Goyang, Republic of Korea \\ 2Department of Psychiatry, Seoul Children's Hospital, Seoul, Republic of Korea \\ ${ }^{3}$ School of Business, Kyung Hee University, Seoul, Republic of Korea \\ ${ }^{4}$ Department of Psychiatry, Graduate School, Kyung Hee University, Seoul, Republic of Korea \\ ${ }^{5}$ Department of Rehabilitation, Hanshin University, Osan, Republic of Korea \\ ${ }^{6}$ Sewon Infant and Child Development Center, Seoul, Republic of Korea \\ ${ }^{7}$ Department of Psychiatry, Konyang University College of Medicine, Konyang University Hospital, Daejeon, Republic of Korea \\ ${ }^{8}$ Department of Psychiatry, Kyung Hee University School of Medicine, Seoul, Republic of Korea
}

\begin{abstract}
Objective We aimed to investigate the current diagnostic incidence, and medical and psychiatric comorbidities of reactive attachment disorder (RAD) using the National Health Insurance Review and Assessment (HIRA) claims data.

Methods To examine the diagnostic incidence, we selected patients who were under 10-year-old and who had at least one medical claim containing a 10th revision of the International Statistical Classification of Diseases and Related Health Problems (ICD-10) code for RAD (F94.1 and F94.2) and who had not been diagnosed in the previous 360 days, from 2010 to 2012. In this study, we used the term 'reactive attachment disorder' representing for both RAD per se and Disinhibited social engagement disorder. Comorbid disorders were categorized according to ICD-10.

Results Among 14,029,571, the total population under 10-year-old during 2010-2012, incident cases of RAD were 736. The mean diagnostic incidence of RAD was 5.25 per 100,000 annually. Language disorders (F80-84) were the most common psychiatric comorbidities in both boys and girls in age groups 0-3 years and 4-6 years, and attention deficit hyperactivity disorder was the most common in both sex aged 7-9 years. In non-psychiatric comorbidities, diseases of the respiratory system (J00-99) were the commonest in both sex in all age groups, and diseases of the digestive system (K00-99) were the next.

Conclusion RAD was very rare in practice and would be disguised as other psychiatric disorders. Children with RAD might have more medical comorbidities than typically developed children.

Psychiatry Investig 2018;15(2):118-123
\end{abstract}

Key Words Reactive attachment disorder, Incidence, Comorbidity.

\section{INTRODUCTION}

Reactive attachment disorder (RAD) is a disorder of social functioning. Although it was first included in the third edition of the Diagnostic and Statistical Manual of Mental Disorders

Received: August 29, 2017 Revised: October 2, 2017

Accepted: November 1, 2017 Available online: November 24, 2017

$\triangle$ Correspondence: Geon Ho Bahn, MD, PhD

Department of Psychiatry, Kyung Hee University School of Medicine, 23 Kyungheedae-ro, Dongdaemun-gu, Seoul 02447, Republic of Korea

Tel: +82-2-958-8542, Fax: +82-2-957-1997, E-mail: mompeian@khu.ac.kr

(c) This is an Open Access article distributed under the terms of the Creative Commons Attribution Non-Commercial License (http://creativecommons.org/licenses/bync/4.0) which permits unrestricted non-commercial use, distribution, and reproduction in any medium, provided the original work is properly cited.
(DSM-III), published in 1980, ${ }^{1}$ there have been almost no systematic studies since then. ${ }^{2}$ In particular, few data exist on the prevalence, sex ratio, or comorbidities of $\mathrm{RAD} .^{3}$ Despite the lack of systematic research, this disorder has been retained in DSM-IV because it presents with unique signs and symptoms that cannot be explained by other conditions. ${ }^{2}$ Based on the combined results of recent studies, the two subtypes of RAD recorded in the DSM-IV appear more as two different disorders than subtypes of the same disease, and to reflect this, they have been classified as the two distinct disorders of RAD and disinhibited social engagement disorder (DSED) in DSM- $5 ., 5$

Up to now, the published results of domestic prevalence on 
RAD were nonexistent, and international results are also very rare. ${ }^{5}$ RAD and DSED are very rare, ${ }^{5}$ with almost no empirical research published, and number of cases have been too few for analysis in DSM-5 field trials. ${ }^{6}$ RAD remains one of the least evidence-based area of DSM and International Statistical Classification of Diseases and Related Health Problems (ICD) nosology. ${ }^{7}$ Though Skovgaard estimated the prevalence to be $0.9 \%$ [ $95 \%$ confidence interval (CI) $0.2-3.4 \%$ ] in 1.5 -year-old children, ${ }^{8} \mathrm{RAD}$ is usually not reported in community sample. ${ }^{4}$ In a deprived population of children aged 6-8 years, the prevalence was $1.40 \%$ (95\% CI 0.94-2.10\%). ${ }^{9}$ Thus, evidence-based epidemiological studies of RAD have severe limitations. While RAD is persistent ${ }^{10}$ and associated with significant psychiatric morbidity, ${ }^{11}$ frequently with cognitive and language delays. Physical problems such as delay in motor, weight and height sometimes occur. ${ }^{4}$ Beyond psychiatric comorbidities in patients with RAD the data for medical comorbidities lack. ${ }^{12}$

The Korean National Health Insurance (NHI) claims database maintained by the Health Insurance Review and Assessment Service (HIRA) is a database containing all medical and prescription drug claim records for the Korean population..$^{13}$ According to the claims data from 2015, approximately 50,490,157 individuals (97.1\%) had National Health Insurance (NHI), while 1,544,267 individuals (2.9\%) had medical aid. ${ }^{14}$ HIRA claims data are a record of national insurance claims by a physician based on patient treatment records, and the diagnostic codes are determined by the clinician after treating the patient. HIRA system adopted Korean standard classification diseases (KCD) diagnostic codes, which is fundamentally based on ICD-10. The diagnostic criteria for RAD in ICD-10 (WHO, 1992) are similar to DSM-IV, but the two subtypes from DSM-IV are classified as different diseases. The inhibited form from DSM-IV is the same as RAD in ICD-10, while the disinhibited form from DSM-IV is similar to disinhibited attachment disorder in ICD- $10 .^{15}$ Therefore, we considered the ICD-10 diagnostic codes F94.1 (RAD of childhood) and F94.2 (disinhibited attachment disorder of childhood) used in the Health Insurance Review and Assessment (HIRA) claims data together as RAD in this study.

Epidemiological data for RAD patients provide basic information for restructuring policies and systems related to infant healthcare and mental health. Given the lack of existing research data, claims data for the national population are an important resource for examining the epidemiology of RAD including diagnosis incidence and comorbidities. The aims of this study were to ascertain the number of children who were diagnosed with RAD, and to determine the types and distribution of comorbidities, psychiatric and non-psychiatric medi$\mathrm{cal}$, in children diagnosed with RAD.

\section{METHODS}

\section{Data source and study population}

We obtained the data from the HIRA from January 1, 2009 to December 31,2013 . The data span 5 years because this is the limit for use of the data for research purposes set by the HIRA. As it is unclear whether RAD occurs in older children, and recommended to be cautions in making decision in children older than 5 years, ${ }^{4,5}$ the subjects in this study were children under 10 years of age: there was no case of newly diagnosed neither as F94.1 nor F94.2 after 10 years old, according to raw data of recently published research using HIRA-National Patient Sample (HIRA-NPS-2011-0082) under 19 years old population. ${ }^{16}$ we only included subject age range up to age 10 .

For analysis, we selected subjects that had at least one claim for either of the ICD-10 17 diagnostic codes F94.1 (RAD of childhood) and F94.2 (disinhibited attachment disorder of childhood), who had not been diagnosed in the previous 360 days. Among the data for 2009-2013, insurance procedures for the 2013 data had not been completed by early 2014 , when the present study commenced, and so these data were excluded. In the final analysis, we used 3 years of National Health Insurance claims data from January 2010 to December 2012.

\section{Process}

The annual incidence was calculated from 2010 to 2012 by dividing the number of newly diagnosed cases of RAD during each year by the number of person-years at risk in the National Health Insurance data set for the same year. We analyzed the subjects' age, sex, type of medical insurance (National Health Insurance/Medical Aid), and type of medical institution visited (e.g., general hospital, mental hospital, or private clinic).

For the comorbidity of psychiatric disorders, the following disorders were categorized based on ICD-10 codes: attention deficit hyperactivity disorder (F90.x), affective disorder (F30. $\mathrm{x}-\mathrm{F} 39 . \mathrm{x})$, somatoform disorder (F45.x-F48.x), mental retardation (F70.x-F79.x), autism spectrum disorder (F84.x), behavioral syndromes associated with physiological disturbances and physical factors (F50.x-F59.x), schizophrenia (F20.xF29.x), anxiety disorder (F40.x-F42.x, F93.x), conduct disorder (F91.x-F92.x), tic disorder (F95.x), developmental disorders of speech and language(F80.x-F83.x), unspecified mental disorder (F99.x), enuresis and encopresis (F98.0 and F98.1), reaction to severe stress and adjustment disorder (F43.x), and others (other F codes). For medical comorbidity, comorbid diseases were categorized according to ICD-10 alphabet group, A to Z. In the analysis of the comorbidities, psychiatric and medical, we compared the diagnosis by gender and age group, 0-3, 4-6, and 7-9 years old. 
This study was approved by the Institutional Review Board (IRB) of Kyung Hee University Hospital, Seoul (IRB No. 201707-030). As claims data were provided anonymously, informed consent was exempted by the IRB.

\section{Statistical analysis}

SAS version 9.3 (SAS Institute Inc., Cary, NC, USA) was used to link and analyze the data. The significance level was set at 0.05 . Descriptive analyses were also performed.

Table 1. Demographic and clinical characteristics of subjects with reactive attachment disorder (RAD) between 2010 and 2012

\begin{tabular}{|c|c|c|}
\hline \multicolumn{2}{|c|}{ Characteristics } & Incident cases, $\mathrm{N}(\%)$ \\
\hline \multicolumn{3}{|l|}{ Age, years } \\
\hline $0-1$ & & $23(3.1)$ \\
\hline 2 & & $119(16.2)$ \\
\hline 3 & & $175(23.8)$ \\
\hline 4 & & $131(17.8)$ \\
\hline 5 & & $85(11.5)$ \\
\hline 6 & & $72(9.8)$ \\
\hline 7 & & $59(8.0)$ \\
\hline 8 & & $41(5.6)$ \\
\hline 9 & & $31(4.2)$ \\
\hline Total & & $736(100)$ \\
\hline Year & $\begin{array}{l}\text { Diagnostic incidence } \\
\quad(\text { Per 100,000) }\end{array}$ & $\begin{array}{l}\text { Total population } \\
\text { aged } 0-9(\mathrm{NSO})\end{array}$ \\
\hline 2010 & 5.39 & $4,769,730$ \\
\hline 2011 & 4.90 & $4,651,969$ \\
\hline 2012 & 5.45 & $4,607,872$ \\
\hline \multicolumn{3}{|l|}{ Sex } \\
\hline Boy & & $522(70.9)$ \\
\hline Girl & & $214(29.1)$ \\
\hline \multicolumn{3}{|l|}{ Region of clinic } \\
\hline Urban & & $244(33.2)$ \\
\hline Rural & & $492(66.8)$ \\
\hline \multicolumn{3}{|l|}{ Types of facility } \\
\hline General hospital & & 355 (48.2) \\
\hline Private clinic & & $377(51.3)$ \\
\hline Others & & $4(0.5)$ \\
\hline \multicolumn{3}{|l|}{ Insurance } \\
\hline National health in & nsurance & $674(91.6)$ \\
\hline Medical aid/lower & r-income group & $62(8.4)$ \\
\hline \multicolumn{3}{|c|}{ Physicians' subspecialty } \\
\hline Psychiatry & & $598(81.2)$ \\
\hline Pediatrics & & $64(8.7)$ \\
\hline General physician & & $60(8.2)$ \\
\hline Others & & $14(1.9)$ \\
\hline
\end{tabular}

NSO: national statistical office

\section{RESULTS}

\section{Demographic characteristics and annual incidence of RAD diagnosis}

The population under 10 years old in Korea was 4,769,730 in 2010, 4,651,969 in 2011, and 4,607,872 in 2012. Among the total population for three years, $14,029,571$, the diagnosis incidence of RAD was 736. Across 2010-2012, newly diagnosed with RAD was the highest for three years old (23.8\%), followed by four years old, then two years old (Table 1). There was a higher proportion of male children $(70.9 \%)$ than that of female $(29.1 \%)$ (Table 1). The percentage of subjects registered for National Health Insurance was $91.6 \%$, whereas $8.4 \%$ had Medical Aid or were in a lower-income group (Table 1). The annual diagnosis incidence of RAD per 100,000 population was 5.25; 5.39 in 2010, 4.90 in 2011, 5.45 in 2012. In every year, the incidence was the highest for children aged 2-3 years and showed a decreasing trend with increasing age (Figure 1).

\section{Psychiatric comorbidities of RAD}

For the distribution of psychiatric comorbidities by age group, developmental disorders of speech and language and autism spectrum disorder (ASD) had higher comorbidity rates in children aged 0-3 than in those aged 4-6 or 7-9 (Figure 2). Children aged 4-6 had higher comorbidity rates than those aged $0-3$ or 7-9 for mental retardation, behavioral and emotional disorders, attention deficit hyperactivity disorder, anxiety disorder, affective disorder, behavioral syndromes associated with physiological disturbances and physical factors, schizophrenia, unspecified mental disorder, and enuresis and encopresis. Children aged 7-9 had higher comorbidity rates than those aged $0-3$ or 4-6 for conduct disorder, reaction to severe stress and adjustment disorder, and tic disorder.

\section{Non-psychiatric comorbidities of RAD}

From the analysis of the non-psychiatric comorbidities of the subjects by age group and sex, non-psychiatric comorbidity

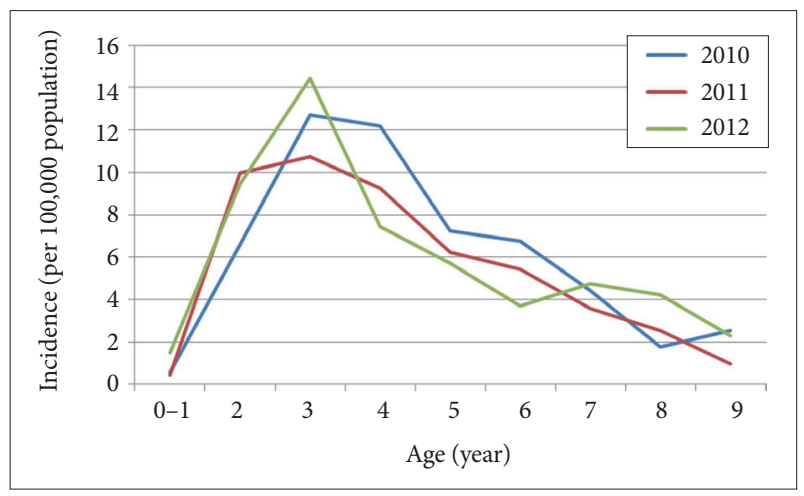

Figure 1. Age-specific annual incidence of reactive attachment disorder between 2010 and 2012 (per 100,000 population). 


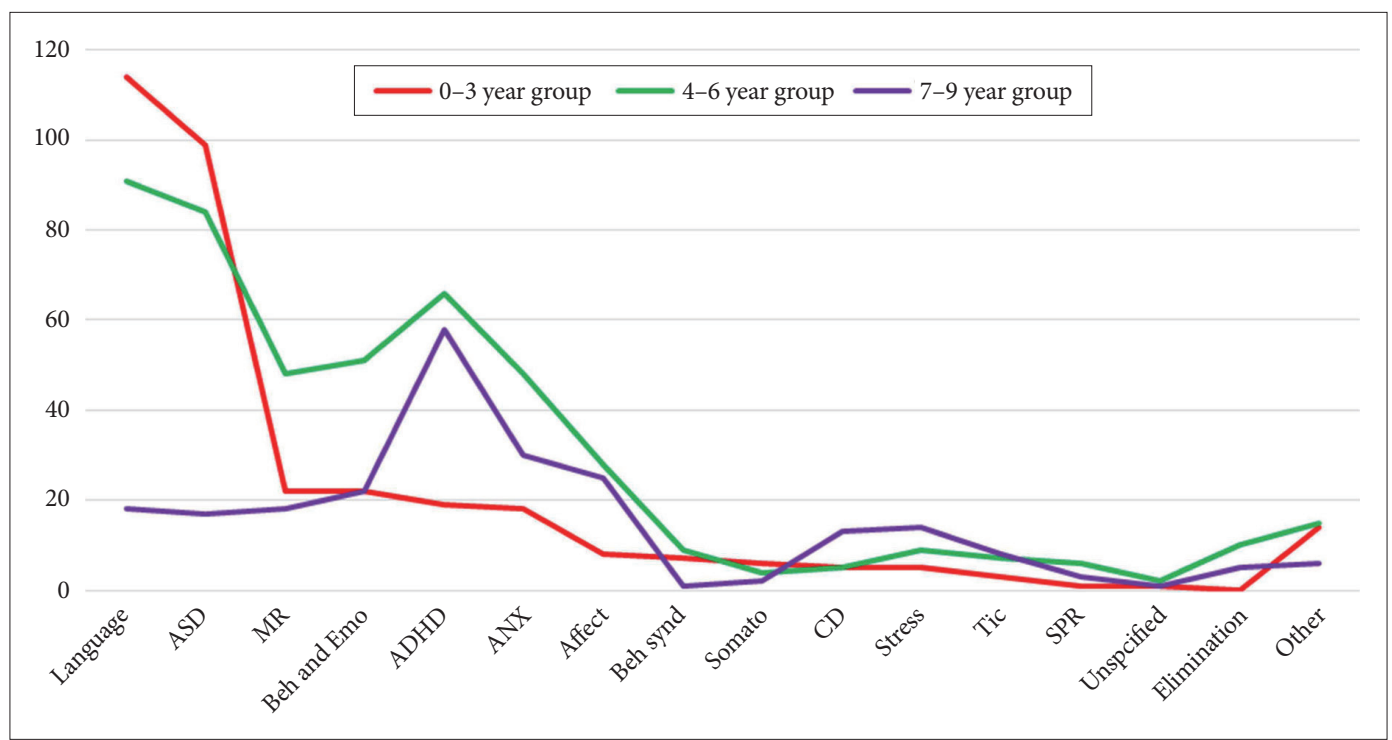

Figure 2. Distribution of psychiatric comorbidities of RAD by age groups $(\mathrm{N})$. RAD: reactive attachment disorder, Language: developmental disorders of speech and language (F80.x-F83.x), ASD: autism spectrum disorder (F84.x), MR: mental retardation (F7x.X), Beh and Emo: behavioral and emotional disorders (F93.8, F98.8, and F98.9), ADHD: attention deficit hyperactivity disorder (F90.X), ANX: anxiety disorder (F40.x-F42.x, F93.0-F93.2), Affect: affective disorder (F3x.X), Beh synd: behavioral syndromes associated with physiological disturbances and physical factors (F5x.x), Somato: somatoform disorders (F45.x-F48.x), CD: conduct disorder (F91.x-F92.x), Stress: reaction to severe stress, and adjustment disorder (F43.x), Tic: Tic disorder (F95.x), SPR: schizophrenia (F2x.x), Unspecified: unspecified mental disorder (F99.x), Elimination: Enuresis and Encopresis (F98.0 and F98.1).

rates were the highest for boys aged $4-6$ followed by boys aged $0-3$, boys aged $7-9$, girls aged $4-6$, girls aged $0-3$, and finally, girls aged 7-9 (Figure 3). When the non-psychiatric comorbidities were analyzed by ICD-10 code, the 10 highest comorbidities were diseases of the respiratory system (J00-J99); diseases of the digestive system (K00-K99); certain infectious and parasitic diseases (A00-B99); diseases of the skin and subcutaneous tissue (L00-L99); symptoms, signs, and abnormal clinical and laboratory findings, not elsewhere classified (R00-R99); diseases of the eye and adnexa (H00-H59); diseases of the ear and mastoid process (H60-H95); injury, poisoning, and certain other consequences of external causes (S00-T98); diseases of the musculoskeletal system and connective tissue (M00-M99); and factors influencing health status and contact with health services (Z00-Z99) (Figure 2).

\section{DISCUSSION}

The annual incidence of RAD in South Korea among children under 10 years of age was 5.25 cases per 100,000 populations. This is lower than the prevalence of $1.4 \%$ reported $^{9}$ in a study of the deprived general population. And another study with highly selected sample, 279 foster children, reported $19.4 \%$ were given RAD diagnosis using web-based diagnostic interview tool by foster parents and teachers. ${ }^{18}$ This discrepancy is thought to be due to differences in methods and biased sample. Minnis et al. ${ }^{9}$ selected children from the deprived sector in the United Kingdom using a scale and semi- structured interview administered to parents, whereas the present study used secondary data from hospital insurance claims from nationwide population. As we used claims data, it is likely that the epidemiology might be different from our results. There are several reasons for this, including the negative social perception of psychiatric diagnoses as well as the terms of insurance products at private insurance companies, which deny insurance for psychiatric diagnoses at medical institutions. As indirect evidence to support the environmental influence as the reason of the big difference of the incidence rate, we can consider the proportion as high as $8.4 \%$ of patients with RAD from medical aid group. Because the proportion of medical aid in general population is $2.9 \%$. This is consistent finding that poverty may influence primary parent-child relationship as one of environmental risk factor. ${ }^{19}$ With regard to common psychiatric comorbidities such as mental retardation, ASD, developmental disorders of speech and language, $\mathrm{ADHD}$, and behavioral and emotional disorders, symptoms from these disorders would overwhelm the feasibility of RAD. If there is not enough awareness of RAD presenting within the general population, there are potential implications leading to exclusive diagnosis and treatment of the comorbid disorder coupled with a lack of recognition of the child's difficulties in forming and maintaining relationship..$^{20}$ It means that RAD is not rare but hard to find. ${ }^{9}$

In this study, developmental disorders of speech and language and autism spectrum disorder showed the highest comorbidity rates in children aged $0-3$. Across all the major di- 


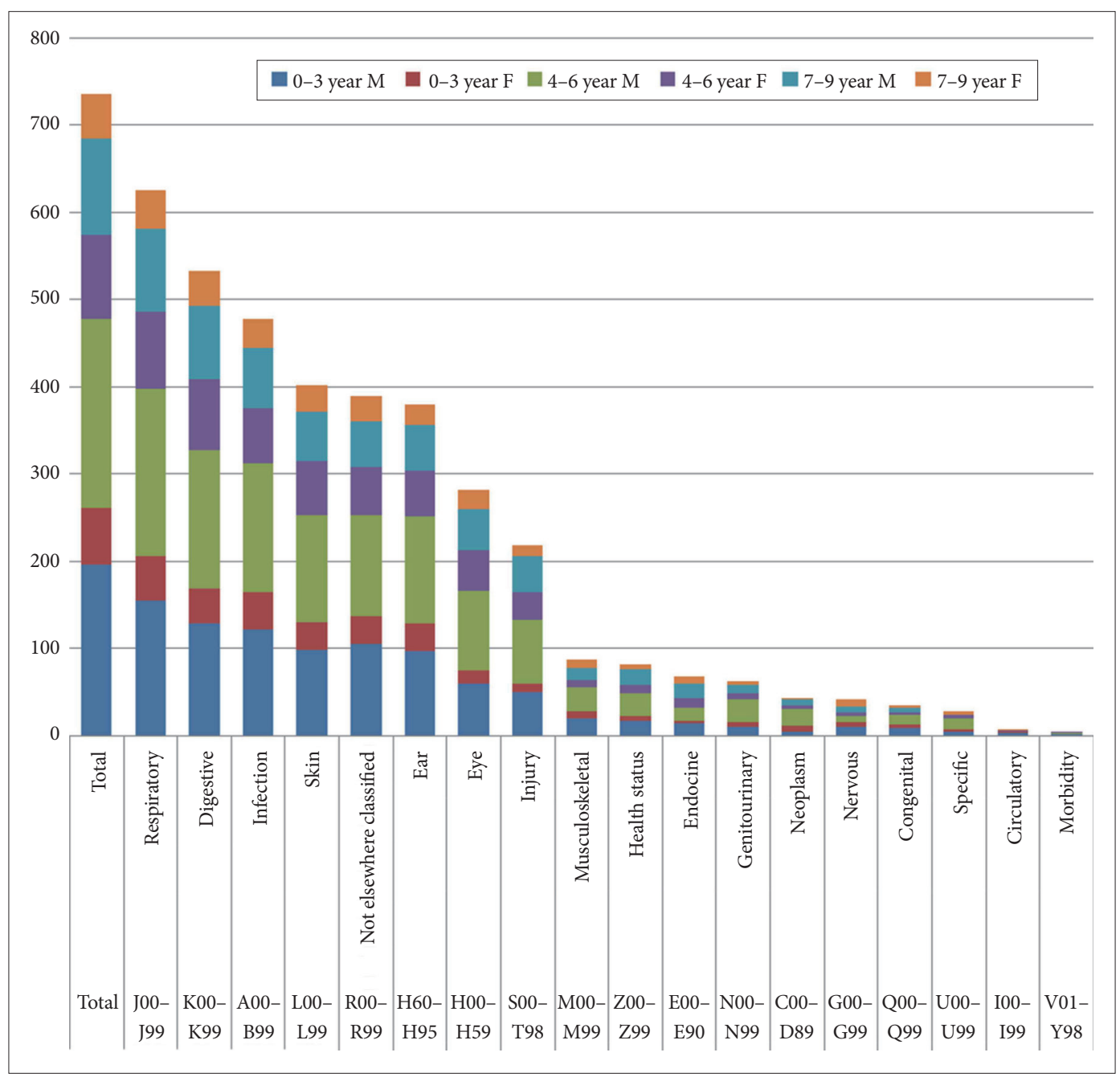

Figure 3. Non-psychiatric comorbidities among children with RAD (N). A00-B99: certain infectious and parasitic disease, C00-D89: neoplasms and diseases of the blood and blood-forming organs, E00-E99: endocrine, nutritional, and metabolic diseases, G00-G99: diseases of the nervous system, $\mathrm{H} 00-\mathrm{H} 59$ : diseases of the eye and adnexa, H60-H95: diseases of the ear and mastoid process, I00-199: diseases of the circulatory system, J00-J99: disease of the respiratory system, K00-K99: disease of the digestive system, L00-L99: diseases of the skin and subcutaneous tissue, M00-M99: diseases of the musculoskeletal system and connective tissue, N00-N99: diseases of the genitourinary system, Q00-Q99: congenital malformations, deformations, and chromosomal abnormalities, R00-R99: symptoms, signs, and abnormal clinical and laboratory findings, not elsewhere classified, S00-T98: injury, poisoning, and certain other consequences of external causes, U00-U99: codes for special purposes, V01-Y98: external causes of morbidity and mortality, Z00-Z99: factors influencing health status and contact with health services, RAD: reactive attachment disorder, F: female, M: male.

agnostic systems such as DSM-5, ICD-10, and Diagnostic classification of mental and developmental disorders of infancy and early childhood (DC:0-5), ${ }^{4}$ RAD cannot be diagnosed as a comorbidity in patients with ASD; however, ASD and RAD are similar with regard to social difficulties, and the lack of relevant research makes it difficult to differentiate between these two diseases. ${ }^{21,22}$ RAD should not be diagnosed in children with autism because of the possibility of mistakenly perceiving autism as RAD. ${ }^{15}$ Meanwhile, "there are insufficient data to date that have addressed whether RAD and pervasive developmental disorder can co-occur" (p.11) and "there are no obvious reasons why a child could not be comorbid for both ASD and RAD" (p.29). Thus, there is an ab- sence of established guidelines or evidence regarding the cooccurrence situation and shared clinical features of RAD and ASD. We consider the present study to be valuable because it uses nationwide data to show the current state of clinicians' diagnostic notions or impressions at the phenomenological level.

The distribution of non-psychiatric symptoms in RAD patients did not show any clear differences in trends by age or sex. Compared with the distribution by disease group among the typical 0-9-year-old population (i.e., the proportion of the typical population with a certain disease) as determined from HIRA annual reports, ${ }^{23} \mathrm{RAD}$ patients showed higher comorbidity rates in all disease groups other than diseases of the respiratory system (J00-J99), diseases of the digestive system 
(K00-K99), and diseases of the eye and adnexa (H00-H59). These results will need to be verified in the future through prospective studies, but we hypothesized that diseases of the digestive system, respiratory system, and eye, which typically increase rapidly in children as they reach school age, may have been neglected in RAD patients, leading to a lower detection rate. Regarding other disease groups, RAD patients seem to have higher comorbidity rates for physical diseases such as skin, infectious, and genetic diseases. Although it is known as a higher comorbidity of failure to thrive $\mathrm{e}^{24}$ or other feeding abnormalities ${ }^{25}$ in children with RAD, we could not investigate about this because comparison of individual diagnosis between study subjects with data of HIRA annual report was not possible. This needs to be verified in the future studies with hospital database.

\section{Limitations}

While this study used hospital claims data, patients who began treatment outside of a hospital were not included, therefore, the results from this study would not reflect the precise incidence of RAD. Hence, this study was based on rather diagnostic codes recorded in claims data, than the standard interviews, diagnostic scales, and laboratory data, diagnostic validity would be issued. Nevertheless, given the global lack of evidence relating to $\mathrm{RAD}$, this study has the strength to examine the diagnosed incidence and psychiatric and medical comorbidity rates for RAD using data recorded by physician representative of the national population.

\section{Clinical implications}

Considering that global interest in ASD has been growing recently and that RAD shares some similar characteristics with ASD, evaluating RAD in more detail not only allows us to accumulate more knowledge and information about RAD itself but can also lead to more appropriate therapeutic interventions by improving the ability to differentiate RAD from ASD and other neurodevelopmental disorders.

\section{Acknowledgements}

This work was supported by the Korea Mental Health R\&D Project, Republic of Korea (Grant No. HM15C1084).

\section{REFERENCES}

1. American Psychiatric Association. Diagnostic and Statistical Manual (DSM-III). Washington, DC: American Psychiatric Association; 1980.

2. Ke H. Korean Textbook of Child Psychiatry. Seoul: Hakjisa; 2014.

3. Sadock BJ, Sadock VA, Ruiz P. Kaplan and Sadock's Synopsis of Psychiatry: Behavioral Sciences/Clinical Psychiatry (11th Ed). Philadelphia, PA: Wolters Kluwer; 2015.

4. ZERO TO THREE (Organization). DC: 0-5TM: Diagnostic Classification of Mental Health and Developmental Disorders of Infancy and Early Childhood. Washington, DC: ZERO TO THREE; 2016.

5. American Psychiatric Association. Diagnostic and Statistical Manual of Mental Disorders (DSM-5 $\left.{ }^{\circledR}\right)$. Washington, DC: American Psychiatric Association; 2013.

6. Regier DA, Narrow WE, Clarke DE, Kraemer HC, Kuramoto SJ, Kuhl EA, et al. DSM-5 field trials in the United States and Canada, Part II: test-retest reliability of selected categorical diagnoses. Am J Psychiatry 2013;170:59-70.

7. Kay C, Green J. Reactive attachment disorder following early maltreatment: systematic evidence beyond the institution. J Abnorm Child Psychol 2013;41:571-581.

8. Skovgaard AM. Mental health problems and psychopathology in infancy and early childhood. An epidemiological study. Dan Med Bull 2010;57:B4193.

9. Minnis H, Macmillan S, Pritchett R, Young D, Wallace B, Butcher J, et al. Prevalence of reactive attachment disorder in a deprived population. Br J Psychiatry 2013;202: 342-346.

10. Gleason MM, Fox NA, Drury S, Smyke A, Egger HL, Nelson CA 3rd, et al. Validity of evidence-derived criteria for reactive attachment disorder: indiscriminately social/disinhibited and emotionally withdrawn/inhibited types. J Am Acad Child Adolesc Psychiatry 2011;50: 216-231.e3.

11. Croft C, Beckett C, Rutter M, Castle J, Colvert E, Groothues C, et al. Early adolescent outcomes of institutionally-deprived and non-deprived adoptees. II: language as a protective factor and a vulnerable outcome. J Child Psychol Psychiatry 2007;48:31-44.

12. Steele JS, Buchi KF. Medical and mental health of children entering the utah foster care system. Pediatrics 2008;122:e703-e709.

13. Hong M, Kwack YS, Joung YS, Lee SI, Kim B, Sohn SH, et al. Nationwide rate of attention-deficit hyperactivity disorder diagnosis and pharmacotherapy in Korea in 2008-2011. Asia Pac Psychiatry 2014;6: 379-385.

14. Hong M, Lee SY, Han J, Park JC, Lee YJ, Hwangbo R, et al. Prescription trends of psychotropics in children and adolescents with autism based on nationwide health insurance data. J Korean Med Sci 2017;32:1687-1693.

15. Zeanah CH, Gleason MM. Reactive Attachment Disorder: a review for DSM-V. Report Presented to the American Psychiatric Association; 2010.

16. Hwangbo R, Chang H, Hong M, Cho S, Bahn GH. The diagnostic distribution of psychiatric disorders among the population under 19 years old: based on the national insurance data. J Korean Acad Child Adolesc Psychiatry 2016;27:139-145.

17. World Health Organization. The ICD-10 Classification of Mental and Behavioral Disorders: Clinical Descriptions and Diagnostic Guidelines. Geneva, Switzerland: World Health Organization; 1992.

18. Lehmann S, Havik OE, Havik T, Heiervang ER. Mental disorders in foster children: a study of prevalence, comorbidity and risk factors. Child Adolesc Psychiatry Ment Health 2013;7:39.

19. Dulcan MK. Dulcan's Textbook of Child and Adolescent Psychiatry. Washington, DC: American Psychiatric Association; 2010.

20. Pritchett R, Pritchett J, Marshall E, Davidson C, Minnis H. Reactive attachment disorder in the general population: a hidden ESSENCE disorder. Scientific World Journal 2013;2013:818157.

21. Sadiq FA, Slator L, Skuse D, Law J, Gillberg C, Minnis H. Social use of language in children with reactive attachment disorder and autism spectrum disorders. Eur Child Adolesc Psychiatry 2012;21:267-276.

22. Davidson C, O'Hare A, Mactaggart F, Green J, Young D, Gillberg C, et al. Social relationship difficulties in autism and reactive attachment disorder: Improving diagnostic validity through structured assessment. Res Dev Disabil 2015;40:63-72.

23. National Health Insurance Service (Korea). 2012 National Health Insurance Statistical Yearbook. National Health Insurance Service. Seoul, Korea: Health Insurance Review \& Assessment Service; 2012.

24. Coolbear J, Benoit D. Failure to thrive: Risk for clinical disturbance of attachment? Infant Ment Health J 1999;20:87-104.

25. Hinshaw-Fuselier S, Boris NW, Zeanah CH. Reactive attachment disorder in maltreated twins. Infant Ment Health J 1999;20:42-59. 